\title{
Aberrant Behavioral and Neurobiologic Profiles in Rodents Exposed to Ethanol or Red Wine Early in Development
}

\author{
Sara De Nicolò • Valentina Carito • Marco Fiore • \\ Giovanni Laviola
}

Published online: 19 June 2014

(C) Springer International Publishing Switzerland 2014

\begin{abstract}
Alcohol intake by women before planning a pregnancy, during pregnancy, and/or during lactation induces brain, behavioral, and physical abnormalities in the fetus, which are known as fetal alcohol spectrum disorders. Thanks to the availability of animal models, some cellular and biochemical processes affecting offspring exposed to ethanol during early development have been extensively investigated. Ethanol effects may be mitigated or increased if offspring also are exposed to either positive or adverse conditions during early life. These environmental conditions may have an impact on neuronal and behavioral development and may increase the risk of developing neuropsychiatric symptoms. Indeed, brain development largely is modifiable by experiences, and neuroplasticity is particularly evident during early phases of life. Molecular mediators for brain development and synaptic plasticity are neurotrophins, such as nerve growth factor and brain-derived neurotrophic factor. Early ethanol exposure not only affects the fetal brain directly, but it also leads to long-lasting consequences that emerge during adulthood and aging. Although the mechanisms responsible for these abnormalities in the fetus are unclear, it is hypothesized that ethanol might damage the central nervous system through oxidative stress processes by increasing free-radical production and decreasing cellular antioxidant ability. Ethanol's effects on animal models have been investigated in detail; however, only few studies have investigated the consequences of early wine consumption. Wine contains several
\end{abstract}

\footnotetext{
S. De Nicolò $\cdot$ V. Carito $\cdot$ M. Fiore $(\bowtie)$

Istituto di Biologia Cellulare e Neurobiologia, CNR, Via Fosso di Fiorano 64, 00143 Roma, Italy

e-mail: marco.fiore@cnr.it

G. Laviola $(\bowtie)$

Istituto Superiore di Sanità, Sect of Behavioral Neuroscience, Dept

BCN, Viale Regina Elena 299, 00161 Roma, Italy

e-mail: giovanni.laviola@iss.it
}

concentrations of ethanol and is consumed largely by Mediterranean populations. We recently compared the consequences of early exposure to ethanol or red wine (at the same ethanol concentration) administered during different stages of development in animal models. Our studies show a series of behavioral and neurologic abnormalities in juvenile, adult, and aged offspring of mice exposed to ethanol. A much smaller number of changes (if any) were evidenced as a consequence of early exposure to red wine. Antioxidant compounds measurable in red wine may have mitigated the toxicity associated with ethanol in the fetus. These "protective" mechanisms in mice pre- and postnatally exposed to red wine, compared with the ethanol group, remain unknown. As a whole, our data should not be interpreted as encouraging women to consume red wine before or during pregnancy and/or lactation. Indeed, in these cases, alcohol intake absolutely must be avoided.

Keywords Animal models · Fetal alcohol syndrome (FAS) . Neurodevelopment $\cdot$ Neuroplasticity $\cdot$ Behavior

\section{Introduction}

During the early 1970 s, the dramatic consequences of gestational ethanol exposure on the newborn were highlighted [1]. Children born to alcoholic mothers were reported to show growth, morphological, and neurodevelopmental abnormalities. This set of alterations was defined collectively as fetal alcohol syndrome. Subsequently, it became evident that the majority of children exposed to ethanol in utero do not exhibit morphological anomalies or growth restrictions, but display significant cognitive and behavioural problems. The term alcohol-related neurodevelopmental disorder was later introduced to label this latter group of children. The term fetal alcohol spectrum disorder (FASD) is now commonly used to 
denote the broad spectrum of morphological and behavioural alterations associated with prenatal alcohol exposure. The most severe FASD is fetal alcohol syndrome (FAS), considered the main cause of nongenetically determined mental retardation [2]. The main physiologic features of FAS are craniofacial anomalies, microcephaly, and neurologic and musculoskeletal abnormalities. Intellectual disability, including severe difficulty in learning and memory, also is a core characteristic of this disorder [3-5]. Ethanol, indeed, is neurotoxic and affects several areas and functions of the brain, including the regions responsible for regulating learning and memory, such as the hippocampus. Currently, the only effective strategy to prevent the development of FASD in offspring is abstinence from alcohol before and during pregnancy and lactation [6]. In Western countries, the incidence of FASD is estimated to be about $1 \%[7,8]$ in newborns, although the actual percentage is unknown.

The development and structure of the brain and behavioral regulation depend largely on gene-environment interaction and early experiences. Ethanol exposure during fetal life and/or adolescence has been shown to increase the risk of developing neuropsychiatric symptoms and/or to increase the propensity to consume alcohol later in life $[9,10]$. To determine how ethanol alters fetal development and identify the consequences in adulthood, many experiments have been conducted in animal models [10]. Indeed, through animal models, it has been possible under strictly regulated conditions to investigate the brain structures and genetic factors involved in ethanol consumption and vulnerability [11]. Those toxic consequences also may be strengthened by adverse life experiences or mitigated by a stimulating and/or protective environment [9].

\section{Brief Note on the Importance of Animal Models in Research}

Animal models of diseases are very important because they mimic biologic processes or pathologies that are difficult to study in humans. These models are possible because the most important biologic mechanisms and processes (e.g., development, regulation, metabolic pathways, genes) are maintained through evolution. Different mammalian species have been used to study FASD, including mice (Mus musculus), rats (Rattus norvegicus), and nonhuman primates (Macaca mulatta). In attempting to understand the behavioral and neurodevelopmental consequences of ethanol exposure, rodents provide several experimental advantages [12, 13]. First, it is difficult to control the timing of developmental alcohol exposure in humans, whereas this timing can be controlled in rodents. Second, rodents can model the human life cycle. On the other hand, results from animal studies must be translatable to humans [9]. Placental transfer of ethanol, as well as plasma ethanol concentrations, is similar in rodents and humans; therefore, translation is expected to be realistic [14]. Findings from animal models have clarified the basic cellular and biochemical mechanisms as well as the consequences of early developmental ethanol exposure [15]. With this framework, a series of recent studies from our group indicate that ethanol exposure in mice during pregnancy has marked effects on nerve growth factor (NGF) and brain-derived neurotrophic factor (BDNF) synthesis and release [16•]. Indeed, growth factor deregulation may be responsible for abnormal development of the central and peripheral nervous systems and also has a main role in associated immune and endocrine pathologies [17•]. Rat offspring born to mothers treated with ethanol during pregnancy are characterized by basal hyperactivity in adulthood and also seem more prone than controls to consume ethanol [18]. Findings from animal studies may have great social impact, because they clearly correlate ethanol exposure during pregnancy with a predisposition to alcohol abuse in the offspring. Other studies in pregnant rats identified two main gestational periods during which alcohol consumption increases the risk of spontaneous alcohol consumption in the adult offspring [9]. Indeed, day 8 of rat gestation has been identified as a crucial period in which only a few doses of ethanol are sufficient to potentiate alcohol consumption in adult rats [19]. It has been proposed that the embryonic stage of development, particularly gastrulation, is highly sensitive to the teratogenic effects of alcohol $[20,21]$. The second crucial period occurs at the end of rat gestation (17-20 embryonic days). During the prenatal and early postnatal phases, offspring are exposed to two effects of ethanol: toxicity and the chemosensory aspects. In this case, low or moderate alcohol doses appear to be enough to predispose offspring to increased ethanol consumption in adulthood [22, 23].

\section{Developmental Neurobiology: Vulnerability and Synaptic Plasticity of Selected Neurotransmitters}

Experimental evidence suggests that pre- and/or postnatal ethanol exposure induces long-lasting alterations in the central nervous system (CNS), leading to neuronal and behavioral disabilities [10]. In recent years, nuclear magnetic resonance imaging has been used to study alcohol-induced cerebral abnormalities in humans exposed to alcohol prenatally. These studies reported brain volume reduction, with the most affected areas being the prefrontal cortex, the cerebellum, the subcortical structures, and the limbic system in general [24]. In addition, alcohol abuse in pregnancy results in dysfunctional regulation of several neurotransmitters, such as serotonin, glutamate, noradrenaline, acetylcholine, histamine [10], and dopamine [9]. Hilakivi [25] suggests that early exposure to ethanol might lead to long-term modification in the monoaminergic system, which is known to mediate high ethanol 
consumption in adults. It also is known that prenatal ethanol exposure may affect the development of the dopaminergic system, and it is related to the characteristic hyperactivity profile often reported in exposed offspring [9]. Abnormalities in the serotoninergic system may lead to adulthood changes in attention level, hyperactivity, and increased impulsive behaviors, including violence and alcoholism, as observed in humans and in primate studies [10].

\section{Effects of Early Ethanol on the Hypothalamic-Pituitary- Adrenal Axis System and Brain Serotonin Function}

In mammals, neurologic development is a gradual process that depends on continuous interaction between the internal and external environments [26]. At the core of this interaction are epigenetic factors that induce functional changes in the nervous system in response to the external environment. The nervous system is modifiable by experiences through an important physiologic process known as "neuroplasticity." Epidemiologic studies suggest that negative episodes, trauma (e.g., violence, childhood neglect), or hostile environmental conditions during early life may have a great impact on brain development and increase the risk for developing neuropsychiatric disorders. In animal models and in humans, it has been observed that adverse events during pre- and/or neonatal life are associated with hypothalamic-pituitary-adrenal (HPA) axis abnormalities and changes in emotionality and elicit behavioral symptoms such as anxiety, depression, and stress disorders, including drug abuse, in adulthood. In this framework, findings from adult rat offspring exposed to ethanol during pregnancy also indicate decreased levels of serotonin (5-HT) and its main metabolite (5-hydroxyindoleacetic acid) [10]. Some effects of reduced brain serotonin are basal changes in locomotion, aggression, and impaired impulse control. The role of early environmental changes appears crucial; indeed, manipulation of dam-pup interaction also may modify the development of the brain 5-HT pathway in the offspring. For example, prolonged maternal separation during the first week of life leads to a decrease in 5-HT transmission in adult rats [10]. Taken together, these findings indicate that the development of the HPA axis and brain 5-HT pathway may be modified either independently or through interaction between fetal exposure to ethanol and changes in the early environment. Furthermore, other important factors, such as gender and genotype, have a role in HPA axis and 5HT pathway development [10, 26].

Primate and rodent experimental models have shown that pre- and postnatal adverse environmental conditions deregulate the organism's stress response, whereas early favorable conditions might reduce the risk of developing stress-related pathologies. In rats, postnatal stimuli, such as parental care, and positive social interactions may lessen adverse HPA axis development [27]. The main source of stimuli for offspring is the mother, and maternal care is connected with both the nervous and behavioral development of pups. Offspring separated from their mothers experience this stress as a real deprivation syndrome, and in this context, levels of adrenocorticotropic hormone (ACTH) and corticosterone are increased [28]. Intriguingly, persistently elevated levels of stress hormones during development have been shown to damage the organization of the CNS. Monkeys have been used to investigate whether early stress and prenatal ethanol are responsible for ACTH levels. In adulthood, these animals have shown elevated cortisol levels, hyperactivity, and an increase in spontaneous ethanol consumption. Findings from these studies corroborate the hypothesis that prenatal ethanol exposure has long-lasting consequences in adult life and that precocious adverse environmental conditions may exacerbate the consequences of early ethanol exposure [10].

\section{Synaptic Plasticity and Brain Development: A Role for Neurotrophins?}

The molecular mediators for brain development and synaptic plasticity are called neurotrophins, the most studied of which are NGF and BDNF [29]. NGF was the first growth factor discovered, by Levi-Montalcini and Hamburger in 1953 [30, 31]. It is released by target tissues and can regulate growth survival and differentiation of several neurons of the peripheral nervous system and CNS [30]. Recently, it was demonstrated that neurotrophins not only sustain neuronal survival and differentiation, but they also play an important role in regulating synaptic plasticity [29]. During CNS development, the expression of neurotrophins and their receptors, TrkA for NGF and TrkB for BDNF [32, 33], are regulated, increased, and specially localized in the hippocampus and cortex, the brain areas involved in the neuronal plasticity phenomena that are the basis of learning and memory mechanisms [34-36]. In fetal growth, abnormal NGF and/or BDNF synthesis can deregulate limbic system development, with long-lasting effects on neuronal connections. Indeed, abnormalities in hippocampus and cortex development, and altered NGF and BDNF levels might be associated with vulnerability to neuropsychiatric pathologies. Epigenetic variables (stress, visual stimuli, drug exposure) can modify neuronal activity and exert an important modulation in the genetic expression of growth factors. It is suggested that modifications in neurotrophin levels during early CNS development might have long-term implications in adulthood [37]. Rodent and nonhuman primate studies have demonstrated that fetal brain development is particularly susceptible to early experiences; this susceptibility is sustained by changes in neurotrophin synthesis, especially that of NGF and BDNF. 


\section{New Experimental Field: A Comparison of Effects Associated with Early Exposure to Ethanol or Red Wine}

Mediterranean populations, particularly those of Italy, Spain, France, and Greece, are well-known for their widespread production and use of wine, especially red wine, which is deeply integrated into their diet and alimentation. Although red wine contains high amounts of ethanol, it also has antioxidant compounds, such as polyphenols [16, 38]. It is interesting to note that the incidence of severe FAS in Italy is much lower than that in other countries [39, 40]. Several hypotheses have been proposed, including a possible protective role of the so-called Mediterranean diet, containing vegetables, cereals, and fruits [41•]; a different genetic susceptibility; and a specific low ethanol concentration. Ceccanti et al. [39] hypothesized that red wine consumption during pregnancy might carry less risk of FASD than other alcoholic beverages that have a similar ethanol concentration, but do not contain effective antioxidant compounds, as is the case for white wine [42].

In this framework, Fiore et al. [16, 38], in a longitudinal analysis, investigated the short- and long-term consequences of ethanol or red wine consumption at the same concentration by offspring during adolescence, adulthood, and aging. The authors used a mouse model to study the persistent neurotoxicity induced by ethanol ( $11 \% \mathrm{vol})$ and red wine (a blend of Montepulciano, Sangiovese, and other Italian cultivars containing total polyphenols, expressed as gallic acid equivalents in milligrams per liter of red wine, of 875 at $11 \%$ vol ethanol) as a consequence of prenatal and postnatal administration. They analyzed NGF and BDNF levels in the offspring's brain (hippocampus, cortex, and cerebellum), as well as cognitive and behavioral parameters, and choline acetyltransferase (ChAT). ChAT is the enzyme responsible for the synthesis of the neurotransmitter acetylcholine, which is implicated in learning and memory processes, and is known to be regulated by NGF [30]. The results showed no differences among groups with regard to reproductive parameters, including pup delivery, mortality, and sex ratio. The body weight of mouse dams was comparable among groups. The most important finding of this study is that early ethanol exposure altered the mouse offspring's NGF and BDNF brain levels. Juvenile mice exposed in utero to ethanol, but not to red wine, showed dramatic changes in NGF and BDNF levels in the hippocampus (Fig. 1), which has a major role in spatial memory and learning processes. In adult mice, early exposure to an ethanol solution altered NGF and BDNF levels in the hippocampus, cerebellum, and cortex. Indeed, aberrant neurotrophin levels also were associated with a reduction in ChAT-positive cells in the septum and nucleus basalis (Fig. 1). Acetylcholine has a major role in behavior; therefore, by measuring performance on tasks such as passive avoidance and plus-maze response (Fig. 1), it was possible to study the altered cognition and emotional behavior in adult mice exposed in utero to ethanol solution or red wine. The authors found changes only in the ethanol group in stretched attend posture score (a measure of risk assessment and anxiety-related behavior) in the plus-maze test and in repeat latency scores in the passive avoidance task (Fig. 1). These parameters indicate that prenatal ethanol use has long-term effects on emotions and behavior.

In our work, we hypothesized that the neurotoxic effect of early ethanol exposure might lead to disruption in memory performance, possibly as the result of neuronal death, synaptic reduction, and general deregulation of the limbic system. Major changes were found in adult mice (90 days). Early exposure to red wine solution had no effect on ChATpositive cells and did not lead to behavioral deficits in adult mice; however, mice exposed to ethanol showed important changes in NGF in the cortex and BDNF in the hippocampus [16•]. Differences in body weight were observed transiently in young mice exposed to ethanol and red wine; these mice weighed less than the control mice. However, differences between groups no longer were evident in adulthood, indicating full recovery of body weight. This effect is comparable to that observed in children with FAS; these children usually weigh less than those without FAS, but may gain weight easily as they grow $[16 \cdot, 39]$.

In another study, Fiore et al. [38] investigated the degree of toxic effects on hepatocyte growth factor (HGF), vascular endothelial growth factor (VEGF), glial cell line-derived neurotrophic factor (GDNF), and NGF levels in mice with early exposure to ethanol $(11 \%)$ or red wine $(11 \%)$. HGF regulates cell motility, morphogenesis, and growth and has a major role in angiogenesis, tumorigenesis, and tissue regeneration. HGF expression is reported to be up-regulated in several types of liver injury, including alcoholic pathologies. Ethanol induces cell necrosis in the liver, and HGF likely potentiates the regenerative process, suggesting a protective role for this factor. VEGF is a mitogen that acts on endothelial and vascular cells, induces angiogenesis, and promotes cell migration. It is suggested that chronic alcohol intake may promote liver neovascularisation and tumorigenesis. VEGF levels are potentiated in chronic and acute hepatitis. GDNF promotes survival and differentiation of dopaminergic cells and inhibits apoptosis [38]. Data show that adult animals treated with ethanol during gestation had an important reduction of GDNF levels in the cortex and a significant increase of VEGF in the liver (Table 1). In this case, ethanol intoxication appeared much more marked for the ethanol group, suggesting that red wine was less toxic than ethanol to the liver. Findings from this study seem to suggest that antioxidant compounds present in red wine 


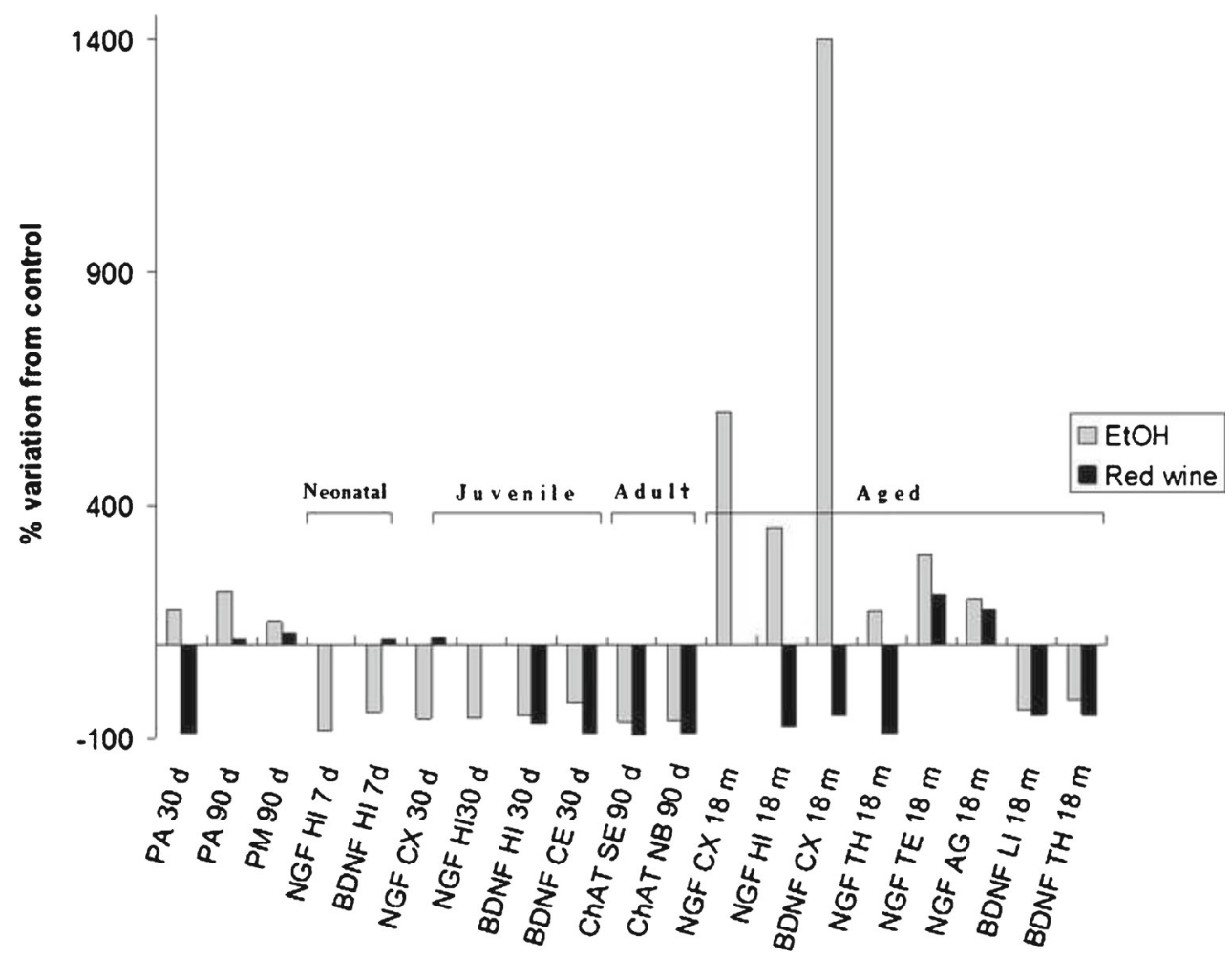

Fig. 1 Behavioural parameters and cognitive domain as measured by passive avoidance performance (PA, RLS "repeated latency scores" ); emotionality domain as measured by plus maze response (PM, SAP "stretched -attended postures") by CD1 male mice at different ages (neonatal 7 day, juvenile 30 day, adult 90 day and aged 18 month-old) early exposed to ethanol or red vine. Neurological parameters, NGF, BDNF and ChAT levels in several brain tissues (hippocampus (HI),

might mitigate the damage caused by ethanol in the fetus; however, the real "protective" mechanism is still unknown.

\section{Long-term Effects of Early Ethanol Exposure}

Fiore et al. [38] also investigated the long-term effects of prenatal ethanol or red wine solution in aged mice (18 months) not only on NGF and BDNF levels, but also on other growth factors, such as HGF and VEGF in the hippocampus, cortex, kidney, and liver, tissues vulnerable to ethanol intoxication. Results indicate that aged mice with early exposure had a dramatic increase in BDNF, HGF, and NGF levels in the frontal cortex and hippocampus compared with controls. In the liver, BDNF levels appeared reduced in both the ethanol and red wine groups. These animals also showed depleted VEGF levels in the liver, whereas in the kidney, BDNF reduction was marked only in mice treated with red wine during gestation.

The effects of ethanol exposure during pregnancy and/or lactation appeared particularly marked in aged mice. Indeed, cortex (CX) and cerebellum (CE), septum (SE) and nuclei basalis (NB)) and peripheral tissues (liver (LI), adrenal glands (AG), thyroid (TH)) the same mice at different ages. Most of parameters illustrated exhibited a significant difference between ethanol vs red wine exposure. Graph redrawn from Fiore $\mathrm{M}$ et al, Toxicology Letters 188 (2009) 208- 213; Ceccanti M et al, Neurobiology of Aging 33 (2012) 359- 367; Ceccanti M, et al, Ann Ist Super Sanità 2013

these data suggest a rapid progression of aging of the brain and liver. NGF potentiation in aged mice may be a sign of precocious brain aging, probably as a result of the accumulation of this neurotrophin in the brain, which might be toxic [38]. HGF also has a major role in aging progression; aged mice exposed to ethanol during early life showed elevated levels in the hippocampus and cortex. Regarding VEGF and BDNF, data show a reduction in the liver during aging. It is proposed that ethanol might stimulate VEGF expression and cancer progression, and ethanol intoxication may promote liver vascularization and tumorigenesis. Therefore, aging is associated with degeneration of several organs and, in the case of early ethanol exposure, with the risk of developing renal or liver damage, which may be potentiated by an increase in VEGF synthesis (Table 1) [43].

In another study, the authors found elevations in NGF and BDNF levels in the thyroid glands of aged mice exposed during development to ethanol solution but not in the group exposed to red wine. In the testis, NGF levels were increased only in the ethanol solution group. In the adrenal glands, elevated NGF levels were found in both the ethanol solution and red wine groups. No changes were observed in the NGF 
Table 1 Hepatocytes growth factor (HGF), vascular endothelial growth factor (VEGF), and glial-derived growth factor (GDNF) in different tissues: liver (LI), hippocampus (HI) and cortex (CX) from juvenile (1 month-old; $1 \mathrm{~m})$ and aged mice (18 month-old; $18 \mathrm{~m})$ exposed early to ethanol or red wine. Most parameters illustrated exhibit significant differences between ethanol vs. red wine exposure. Table redrawn from Fiore $\mathrm{M}$ et al, Toxicology Letters 188 (2009) 208- 213; Ceccanti M et al, Neurobiology of Aging 33 (2012) 359- 367; Ceccanti M, et al, Ann Ist Super Sanità 2013

\begin{tabular}{llll}
\hline & & \multicolumn{2}{l}{$\%$ variation from control } \\
\cline { 3 - 4 } & & Red wine & EtOH \\
\hline Juvenile & HGF L1 1 m & 133.3 & 166.6 \\
& HGF CX 1 m & 162.5 & 32.5 \\
& HGF HI 1 m & 147 & 11.7 \\
& VEGF LI 1 m & 280 & 180 \\
& VEGF CX 1 m & 66.6 & 50 \\
& VEGF HI 1 m & 130 & 110 \\
& GDNF LI 1 m & 77.7 & 122.2 \\
Aged & GDNFCX 1 m & 100 & 71.4 \\
& GDNF HI 1 m & 130 & 80 \\
& HGF CX 18 m & 2.5 & 960 \\
& HGF HI 18 m & 350 & 75 \\
HGF LI18 m & 68 & 76 \\
HGF KI 18 m & 1.8 & 83.3 \\
VEGF CX 18 m & 150 & 650 \\
VEGF HI 18 m & 125 & 350 \\
VEGF LI 18 m & 43.75 & 25 \\
VEGF KI 18 m & 53.8 & 69.2 \\
\hline
\end{tabular}

and BDNF receptors, TrkA and TrkB, respectively, or in the phosphorylated receptors (phospho-TrkA and phospho-TrkB) in any of the tissues examined. The consequences of altered levels of neurotrophic factors in the endocrine glands remain unknown. These experiments indicate that prenatal exposure to ethanol may alter neurotrophin synthesis, release, and utilization by cells of target organs [17].

\section{Red Wine and Polyphenols}

Data show that mice exposed only to red wine did not display the alterations in brain morphology or disruption in cognitive and learning performances observed in the ethanol group [16•, 38]. Most likely, this "protective brain effect" is related to the presence of polyphenols in red wine. The scientific community's attention now is focused on these compounds, organic molecules present in plants and plant-derived products. For example, flavonoids are present in tea (Camellia sinensis L.) and blueberries (Vaccinium myrtillus L.), and catechins in cocoa
(Theobroma cacao L.) and dark chocolate, whereas, in the grapevine (Vitis vinifera L.) and red wine, tannins (resveratrol) are the main antioxidants. Polyphenols are molecules that scavenge free-radicals, preventing cardiovascular diseases by regulating plasma levels of oxidized low-density lipoprotein. Polyphenols also play a role in anti-inflammatory effects, the most important of which are their ability to prevent oxidative damage, which is known to be induced by ethanol in the brain and many other tissues [44].

\section{Conclusions}

Alcohol consumption by women before and/or during pregnancy and/or lactation is known to result in FASD in exposed offspring. Several animal models have been used to investigate short, medium and long-term consequences of exposure to ethanol. During development, the brain is easily modifiable by experience, and this important physiologic process, known as neuroplasticity, is particularly marked during early phases. Several studies have demonstrated that ethanol consumption during pregnancy can affect NGF and BDNF levels, especially in the hippocampus and cortex, leading to unique neurologic and behavioral disorders later in life. Epidemiological reports indicate that the prevalence of FASD in Mediterranean areas is lower than that of the rest of the world. This has led to the speculation that the low rate of prevalence may be a by-product of diet (cereals, olive oil, fruits, vegetables, etc.); high concentrations of antioxidant substances in red wine, such as polyphenols and anthocyanins; or a reduced genetic susceptibly to alcohol toxicity. The studies reviewed here demonstrate that the antioxidants in red wine might mitigate the alterations induced in the fetus or neonate by ethanol, even if it contains the same amount of ethanol as other alcoholic beverages. Importantly, however, our data must not be interpreted as encouragement to drink or abuse red wine. In particular, alcohol must absolutely be avoided by women while planning a pregnancy, during pregnancy, and during lactation.

Acknowledgments Sara De Nicolò, Valentina Carito, and Marco Fiore were supported by CNR. Giovanni Laviola was supported by ISS.

\section{Compliance with Ethics Guidelines}

Conflict of Interest Sara De Nicolò, Valentina Carito, Marco Fiore, and Giovanni Laviola declare that they have no conflict of interest.

Human and Animal Rights and Informed Consent This article does not contain any studies with human or animal subjects performed by any of the authors 


\section{References}

Papers of particular interest, published recently, have been highlighted as:

- Of importance

1. Jones KL, Smith DW. Recognition of the fetal alcohol syndrome in early infancy. Lancet. 1973;302:999-1001.

2. Abel EL, Sokol RJ. Incidence of fetal alcohol syndrome and economic impact of FAS-related anomalies. Drug Alcohol Depend. 1987;19(1):51-70.

3. Mancinelli R, Ceccanti M, Laviola G. Fetal alcohol spectrum disorders (FASD): from experimental biology to the search for treatment. Neurosci Biobehav. 2007;31:165-7.

4. Ikonomidou C. Triggers of apoptosis in the immature brain. Brain Dev. 2009;31:488-92.

5. Manzo-Avalos S, Saavedra-Molina A. Cellular and mitochondrial effects of alcohol consumption. Int J Environ Res Public Health. 2010;7:4281-304.

6. Mancinelli R, Vitali M, Ceccanti M. Women, alcohol and the environment: an update and perspectives in neuroscience. Funct Neurol. 2009;24(2):77-81.

7. Sampson PD, Streissguth AP, Bookstein FL, Little RE, Clarren SK, Dehaene P, et al. Incidence of fetal alcohol syndrome and prevalence of alcohol-related neurodevelopmental disorder. Teratology. 1997;56(5):317-26.

8. O'Leary CM. Fetal alcohol syndrome: diagnosis, epidemiology, and developmental outcomes. J Paediatr Child Health. 2004;40:2-7.

9. Chotro $\mathrm{MG}$, Arias $\mathrm{C}$, Laviola G. Increased ethanol intake after prenatal ethanol exposure: studies with animals. Neurosci Biobehav Rev. 2007;31(2):181-91.

10. Macrì S, Spinelli S, Adriani W, Dee Higley J, Laviola G. Early adversity and alcohol availability persistently modify serotonin and hypothalamic-pituitary-adrenal-axis metabolism and related behavior: what experimental research on rodents and primates can tell us. Neurosci Biobehav Rev. 2007;31(2):172-80.

11. Allison MJ, Ryabinin A, Ryabinin AE. Biological contribution to social influences on alcohol drinking: evidence from animal models. Int J Environ Res Public Health. 2010;7:473-93.

12. Becker HC, Lopez MF, Doremus-Fitzwater TL. Effects of stress on alcohol drinking: a review of animal studies. Psychopharmacology. 2011;218(1):131-56.

13. Schwandt ML, Lindell SG, Chen S, Higley JD, Suomi SJ, Heilig $\mathrm{M}$, et al. Alcohol response and consumption in adolescent rhesus macaques: life history and genetic influences. Alcohol. 2010;44(1): 67-80.

14. Painter A, Andrew D, Burd L. Fetal alcohol spectrum disordersimplications for child neurology, part I: prenatal exposure and dosimetry. J Child Neurol. 2012;27(2):258-63.

15. Aloe L. Alcohol intake during prenatal life affects neuroimmune mediators and brain neurogenesis. Ann Ist Super Sanità. 2006;42(1):17-21

16. Fiore M, Laviola G, Aloe L, di Fausto V, Mancinelli R, Ceccanti M. Early exposure to ethanol but not red wine at the same alcohol concentration induces behavioral and brain neurotrophin alterations in young and adult mice. Neurotoxicol. 2009;30(1):59-71. This article demonstrates that in mice, the effects of prenatal ethanol are more marked than those of red wine exposure.

17. Ceccanti M, De Nicolò S, Mancinelli R, Chaldakov G, Carito $\mathrm{V}$, Ceccanti M, et al. NGF and BDNF long-term variations in the thyroid, testis and adrenal glands of a mouse model of fetal alcohol spectrum disorders. Ann Ist Super Sanità. 2013;49(4): 383-90. This article demonstrates how prenatal ethanol alters the endocrine system and how its effects are maintained during aging.

18. Bond NW, DiGiusto EL. Effects of prenatal alcohol consumption on shock avoidance learning in rats. Psychol Rep. 1976;46:163-5.

19. Molina JC, Hoffmann H, Spear LP, Spear NE. Sensorimotor maturation and alcohol responsiveness in rats prenatally exposed to alcohol during gestational day 8. Neurotoxicol Teratol. 1987;9(2): 121-8.

20. Godin EA, O'Leary-Moore SK, Khan AA, Parnell SE, Ament JJ, Dehart DB, et al. Magnetic resonance microscopy defines ethanolinduced brain abnormalities in prenatal mice: effects of acute insult on gestational day 7. Alcohol Clin Exp Res. 2010;34(1):98-111.

21. Sulik KK, Johnston MC. Sequence of developmental alterations following acute ethanol exposure in mice: craniofacial features of the fetal alcohol syndrome. Am J Anat. 1983;166(3):257-69.

22. Dominguez HD, Lopez MF, Chotro MG, Molina JC. Perinatal responsiveness to alcohol's chemosensory cues as a function of prenatal alcohol administration during gestational days 17-20 in the rat. Neurobiol Learn Mem. 1996;65(2):103-12.

23. Arias $\mathrm{C}, \mathrm{Chotro} \mathrm{MG}$. Increased preference for ethanol in the infant rat after prenatal ethanol exposure, expressed on intake and taste reactivity tests. Alcohol Clin Exp Res. 2005;29(3):337-46.

24. Lebel C, Roussotte F, Sowell ER. Imaging the impact of prenatal alcohol exposure on the structure of the developing human brain. Neuropsychol Rev. 2011;21:102-18.

25. Hilakivi L. Effects of prenatal alcohol exposure on neonatal sleepwake behaviour and adult alcohol consumption in rats. Acta Pharmacol Toxicol. 1986;59:36-42.

26. Becker KL, Walton-Moss B. Detecting and addressing alcohol abuse in women. Nurs Pract. 2001;26:13-6.

27. Laviola G, Rea M, Morley-Fletcher S, Di Carlo S, Bacosi A, De Simone R, et al. Beneficial effects of enriched environmental on adolescent rats from stressed pregnancies. Eur J Neurosci. 2004;20: 1655-64.

28. Solzaman W, Abbott DH. Hormonal and behavioral responses to stress in lactating and non-lactating female common marmosets. Physiol Behav. 2011;104:446-53.

29. McAllister AK, Katz LC. Neurotrophins and synaptic plasticity. Annu Rev Neurosci. 1999;22:295-318.

30. Levi-Montalcini R. Nerve growth factor thirty-five years later. EMBO J. 1987:6:1145-54.

31. Aloe L, Alleva E, Bohm A, Levi-Montalicini R. Aggressive behavior induces release of nerve growth factor from mouse salivary gland into the bloodstream. Neurobiology. 1986;83:6184-7.

32. Huang EJ, Reichardt LF. Trk receptors: roles in neuronal signal transduction. Annu Rev Biochem. 2003;72:609-42.

33. Davis MI. Ethanol-BDNF interactions: still more questions than answers. Pharmacol Ther. 2008;118:36-57.

34. Aloe L, Alleva E, Fiore M. Stress and nerve growth factor: findings in animal models and humans. Pharmacol, Biochem Behav. 2002;73:159-66.

35. Hauser SR, Getachew B, Taylor ER, Tizabi Y. Alcohol induced depressive-like behavior is associated with a reduction in hippocampal BDNF. Pharmacol, Biochem Behav. 2011;100(2):253-8.

36. Allen SJ, Watson JJ, Shoemark KD, Barua NU, Patel NK. GDNF, NGF and BDNF as therapeutic options for neurodegeneration. Pharmacol Ther. 2013;138(2):155-75.

37. Cirulli F, Francia N, Berry A, Aloe L, Alleva E, Suomi SJ. Early life stress as a risk factor for mental health: role of neurotrophins from rodents to non-human primates. Neurosci Biobehav Rev. 2009;33(4):573-85.

38. Fiore M, Mancinelli R, Aloe L, Laviola G, Sornelli F, Vitali M, et al. Hepatocyte growth factor, vascular endothelial growth factor, glial cell-derived neurotrophic factor and nerve growth factor are differentially affected by early chronic ethanol or red wine intake. Toxicol Lett. 2009;188(3):208-13. 
39. Ceccanti M, Spagnolo A, Tarani L, Attilia ML, Chessa L, Mancinelli R. Clinical delineation of fetal alcohol spectrum disorders (FASD) in Italian children: comparison and contrast with other racial/ethnic groups and implications for diagnosis and prevention. Neurosci Biobehav. 2007;31:270-7.

40. Fiorentino D, Coriale G, Spangolo PA, Prastaro A, Attilia ML, Mancinelli R, et al. Fetal alcohol syndrome disorders: experience on the field. The Lazio study preliminary report. Ann Ist Super Sanità. 2006;42(1):53-7.

41. De Nicoló S, Tarani L, Ceccanti M, Maldini M, Natella F, Vania A, et al. Effects of olive polyphenols administration on nerve growth factor and brain-derived neurotrophic factor in the mouse brain. Nutrition. 2013;4:681-7. This is a very important article showing that polyphenols in extra virgin olive oil influence $N G F$ and $B D N F$ synthesis.

42. Aloe L, Tirassa P. The effect of long-term alcohol intake on brain NGF-target cells of aged rats. Alcohol. 1992;9(4):299304.

43. Ceccanti M, Mancinelli R, Tirassa P, Laviola G, Rossi S, Romeo M, et al. Early exposure to ethanol or red wine and long-lasting effects in aged mice. A study on nerve growth factor, brain-derived neurotrophic factor, hepatocyte growth factor, and vascular endothelial growth factor. Neurobiol Aging. 2012;33(2):359-67.

44. Joseph SV, Edirisinghe I, Burton-Freeman BM. Berries: antiinflammatory effects in humans. J Agric Food Chem. 2014;62(18):3886-903. 